Although simple excision has been successful in some cases, ${ }^{6}$ recurrences have been reported in others. ${ }^{35}$ The latter authors recommend primary keratectomy with insertion of a lamellar graft to lessen the risk of recurrence and provide a better cosmetic result.

1 Sonoda T, Hashimoto H, Enjoji M. Juvenile xanthogranuloma: clinicopathological analysis and immunohistochemical study of 57 patients. Cancer 1985; 56: 2280-6.
2 Zimmerman LE. Ocular lesions of juvenile xanthogranuloma.

Am 7 Ophthalmol 1965; 60: 1011-35.
3 Collum LMT, Mullaney J. Adult limbal xanthogranuloma. $\mathrm{Br} \mathcal{F}$ Ophthalmol 1984; 68: 360-3.

4 Lewis JR, Drummond GT, Mielke BW, Hassard DT, Astle WF. Juvenile xanthogranuloma of the corneo-scleral limbus. Can f Ophthalmol 1990; 25: 351-4.

5 Collum LM, Power WJ, Mullaney J, Farrell M. Limbal xanthogranuloma. F Paediatr Ophthalmol Strabismus 1991; 28: 157-9.

6 Kaufman JG, Driebe WT, Hamed LM, Margo CE. Juvenile xanthogranuloma of the corneo-scleral limbus. Ophthalmic Surg 1993; 24: 428-30.

\title{
Fundus albipunctatus-like lesions in juvenile retinoschisis
}

\author{
Mary J van Schooneveld, Yozo Miyake
}

The Netherlands

Ophthalmic Research

Institute, Department of

Ophthalmogenetics,

Postbox 12141, 1100 AC

Amsterdam, the

Netherlands

$M \mathrm{~J}$ van Schooneveld

Nagoya University

School of Medicine,

65 Tsurumai-Cho,

Showa-Ku, Nagoya 466,

Japan

Y Miyake

Correspondence to:

Mary J van Schooneveld.

Accepted for publication

11 April 1994

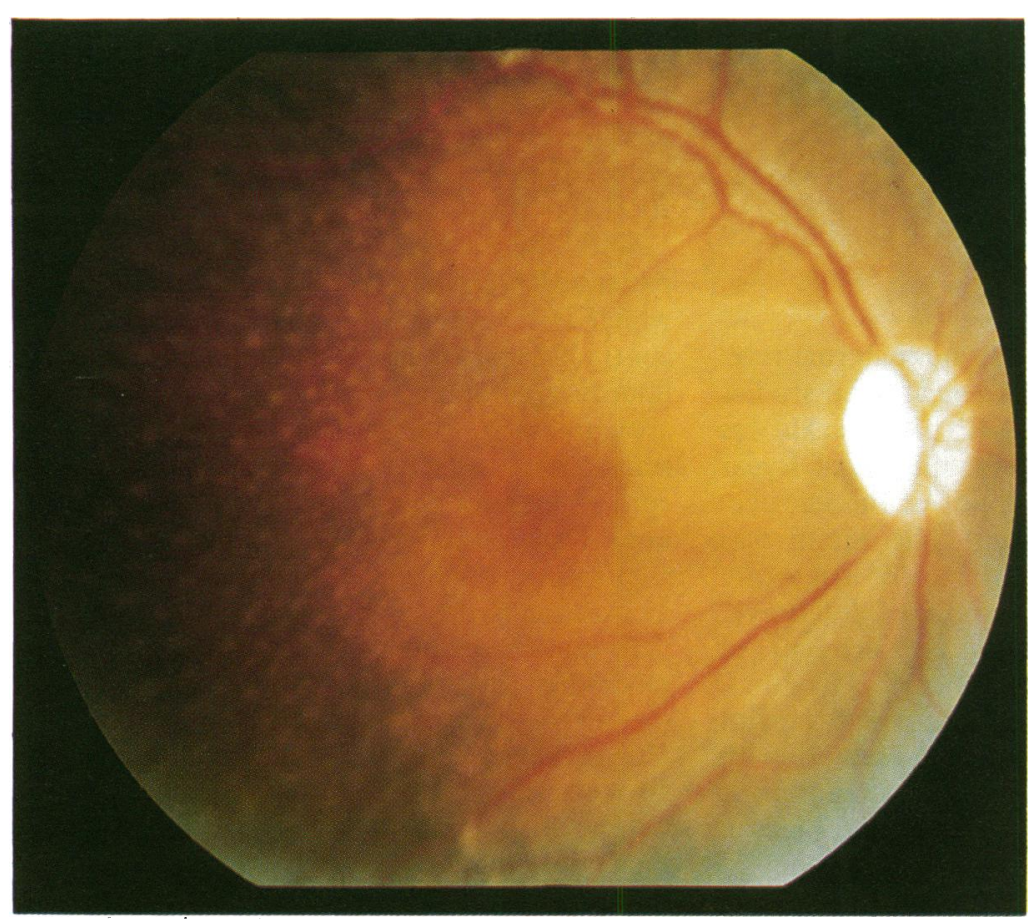

Figure $1 A$ Posterior pole in the right eye of case 1 showing cystic maculopathy and fundus albipunctatus-like lesions.

Juvenile retinoschisis (XRS) is an $\mathrm{X}$ linked vitreoretinopathy characterised mainly by typical maculopathy - namely, foveal schisis. However, the ophthalmoscopic findings may be heterogeneous, varying from a rather subtle cystic maculopathy to what may resemble an old and extensive chorioretinitis. ${ }^{1}$ Apart from the maculopathy and peripheral retinoschisis, glistening golden peripheral areas sometimes resembling the Mizuo phenomenon, dendriform lesions, neovascularisation (peripheral and of the

\section{Case reports}

CASE 1

A 10-year-old Japanese boy was diagnosed as having XRS at the age of 11 months. His maternal grandfather was also known to have this condition.

Visual acuity was 20/200 right eye with $\mathrm{S}+4 \cdot 5=\mathrm{C}+1 \times 55^{\circ}$ and $20 / 100$ left eye with $\mathrm{S}+3=\mathrm{C}+1 \cdot 25 \times 110^{\circ}$. Both fundi showed the typical star-shaped configuration in the macula and peripheral retinoschisis inferiorly. Furthermore multiple small white flecks were scattered in the temporal posterior pole (Fig 1A and 1B). These bilateral white flecks seemed to be located in the retina and not in the pigment epithelium.

The Ganzfeld electroretinogram (ERG) showed markedly reduced scotopic (rod) responses $(30-50 \%$ of normal value) and even more reduced photopic (cone) responses (20$25 \%$ of the normal value) and a negative-type ERG with a single bright flash stimulus. The oscillatory potentials were absent (Fig 2).

Visual fields showed bilateral superior loss corresponding to the schisis areas. There were no complaints of night blindness. In the 62-year-old grandfather the small flecks were absent. 


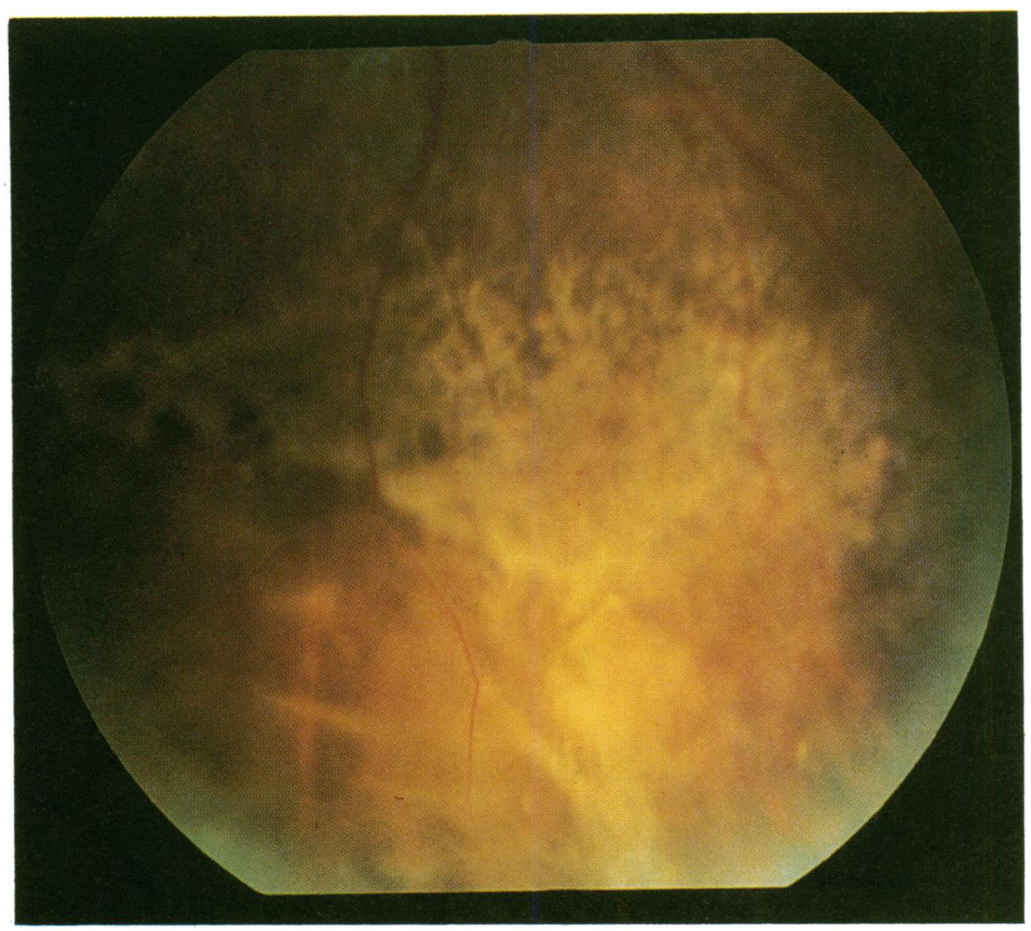

Figure 1B Peripheral retinoschisis and opacified vessels in case 1 .

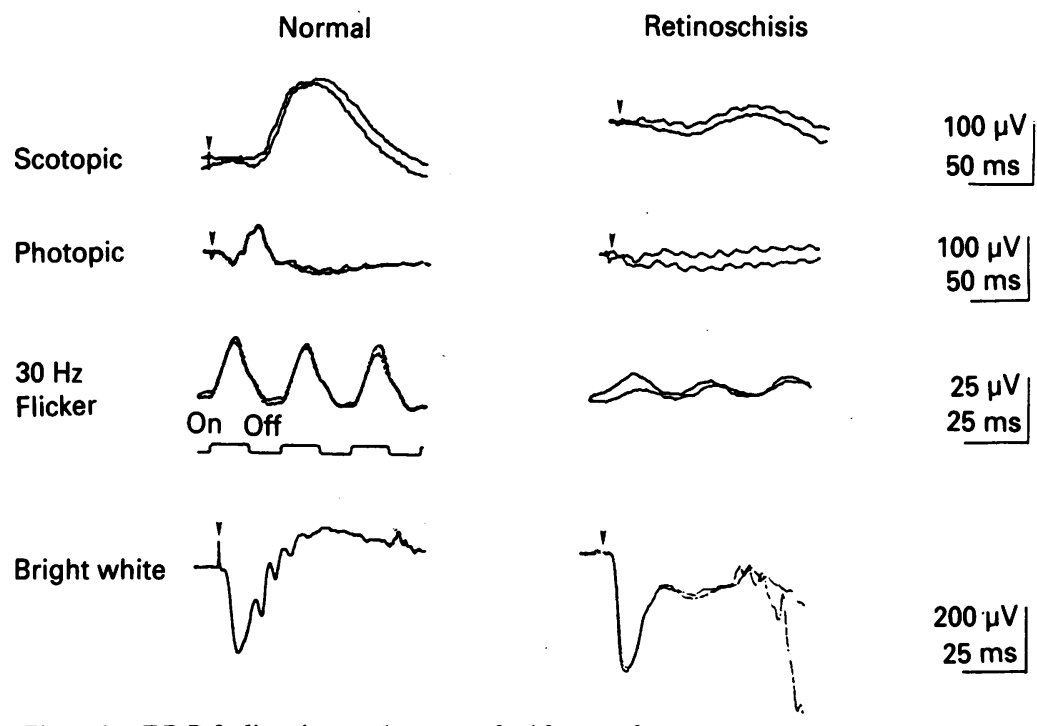

Figure 2 ERG findings in case 1 compared with normal.

CASE 2

A 13-year-old white male had no maternal relatives with known XRS and genealogical studies revealed no link with other XRS patients. He was seen by an ophthalmologist at the age of 1 year because of a convergent squint of the left eye. Occlusion was not tolerated. The squint was operated on and at the age of 7 low visual acuity was noted: 20/60 right eye and 20/200 left eye.

$\mathrm{He}$ was first seen at the department of ophthalmogenetics at the age of 8 . His fundi showed impressive cystic maculopathy with bilateral peripheral inferior retinoschisis with obliterated vessels in the right eye and a fibrotic sea fan with some exudates and haemorrhages in the left eye. Both posterior poles showed numerous small white flecks (Fig 3A). Fluorescein angiography showed transmission defects in the pigment epithelium creating a bull's eye maculopathy, but no pattern corresponding to the albipunctatus-like flecks (Fig 3B). The ERG was negative with almost absent $b$-waves and the EOG had an L/D ratio of only 1.16 and 1.08 respectively. Visual fields disclosed superior defects caused by peripheral schisis. Colour vision measured with the 28 hue test was almost normal (some minor mistakes), but of the Ishihara test only 12 plates (including the test plate) were correctly recognised. Subjectively there was no night blindness.

During the 4.5 years of follow up visual acuity remained stable at 20/60 right eye and 20/200 left eye, both with $S+4 \cdot 5$.

\section{Comment}

In both patients the cystic maculopathy, the peripheral retinoschisis, and the negative configuration of the ERG are characteristic for juvenile retinoschisis. In addition, the maternal grandfather of case 1 was known to have this condition. However, multiple small white flecks were associated with these findings. These small punctate flecks are strongly reminiscent of fundus albipunctatus, including the typical radial orientation relative to the fovea in the first patient (Fig 1A). Fundus albipunctatus is a stationary congenital night blinding disorder characterised by typical white punctate fundus lesions and an abnormal slow regeneration of visual pigment. Although the visual function is stationary, the appearance and number of dots may change over time. ${ }^{3}$ Visual acuity should be normal or near normal. Differentiation from retinitis punctata albescens, a condition belonging to the group of tapetoretinal dystrophies, may occasionally be difficult and is made mainly by the progressive character and visual field loss.

Recently, fundus albipunctatus in combination with cone dystrophy was described by Miyake et $a l .{ }^{4}$ These patients were clearly different from the two patients described in this report, in that the first had progressive loss of central vision starting in adulthood (visual acuity having been normal before), bull's eye maculopathy, no peripheral retinoschisis, and severely reduced photopic ERGs but no negative type ERG.

With fluorescein angiography our second patient also showed a bull's eye maculopathy, which is not typical for XRS, but also is not contradictory. In early stages of XRS often no fluoroangiographic abnormalities in the macular area are seen, but in later stages all kind of transmission defects of the retinal pigment epithelium may be encountered. The albipunctatus flecks could not be traced down in the angiogram, which corresponds to their supposed localisation in the superficial retina.

In most cases of XRS the EOG is normal or moderately reduced, while in our second patient the EOG showed hardly any light increase. However, very abnormal or even flat type EOGs also occur in XRS. ${ }^{5}$ As widespread window defects of the retinal pigment epithelium can be seen on the fluorescein angiogram, the pathological EOG in this patient is explicable. Boisdequin et al do not mention EOG results in their four patients, and so we do not know for the 


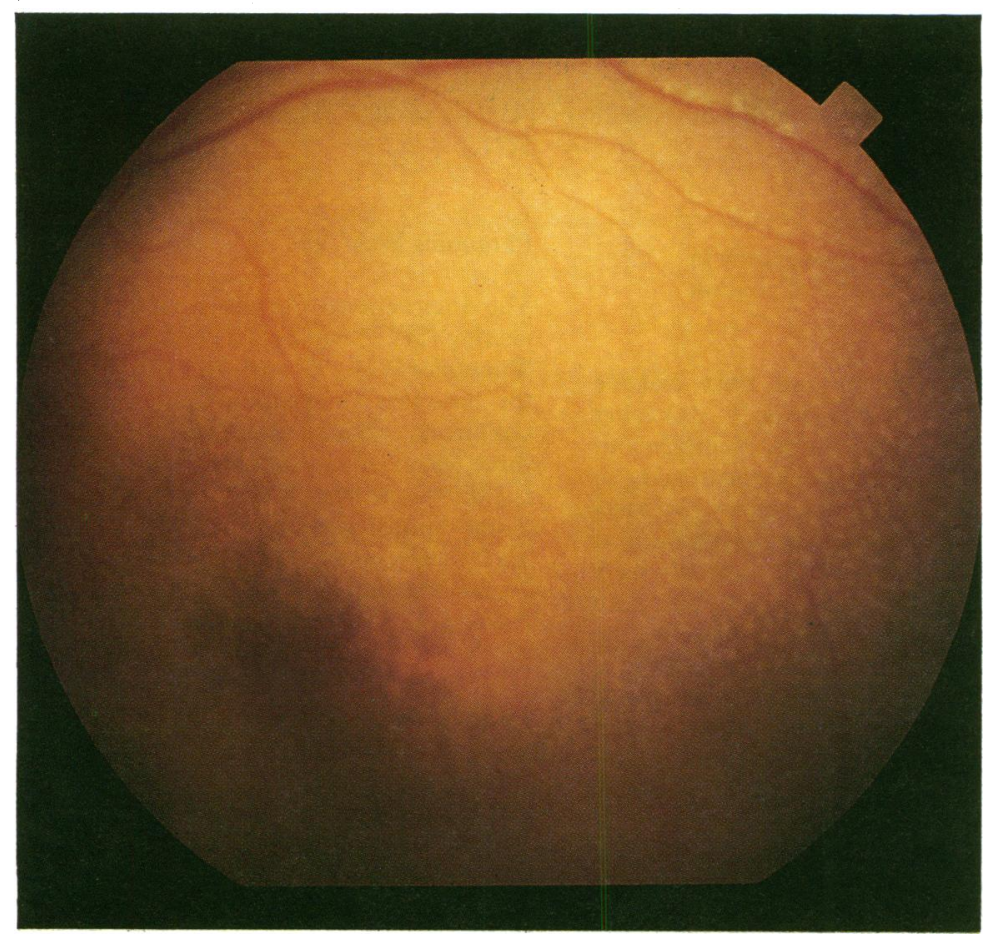

Figure 3A Posterior pole of left eye of case 2 at age 8 years, showing cystic maculopathy and fundus albipunctatus-like lesions.

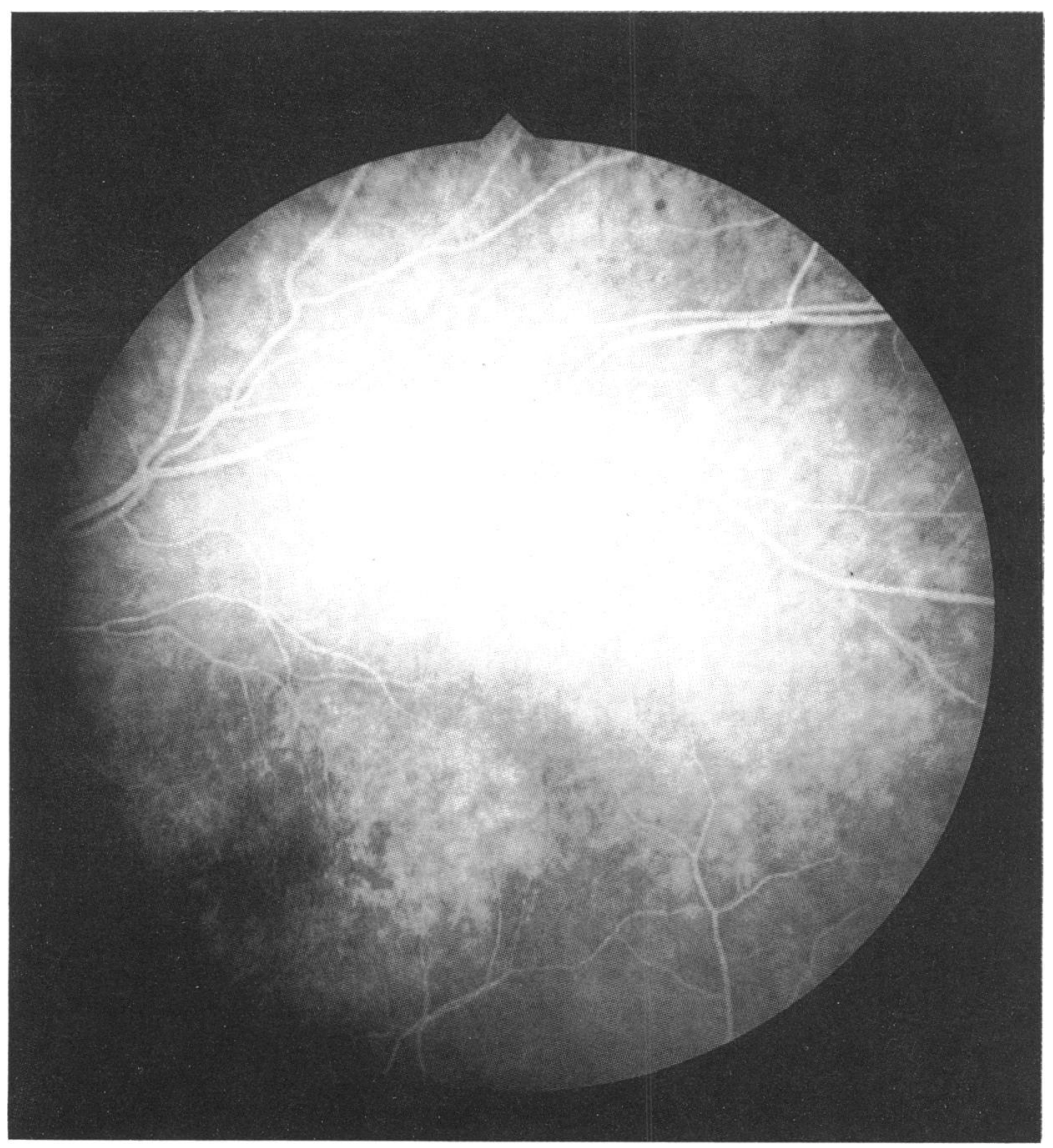

Figure 3B Fluorescein angiogram of the left eye of case 2 showing hyperfluorescence resembling bull's eye maculopathy, but no sign of the punctate lesions. moment whether XRS with fundus albipunctatus-like flecks is associated with an extra low $\mathrm{L} / \mathrm{D}$ ratio of the EOG.

To our knowledge the fundus albipunctatuslike aspect in XRS has not yet been described, apart from in the paper by Boisdequin et al. ${ }^{2}$ In four patients out of nine with XRS described by Bolsdequin et al, the white dots were thought to belong to an early stage of the evolution of the maculopathy where the dots represent 'piliers' (little strands in the superficial retina) comparable with those in acquired retinoschisis. (The photograph on page 58 in their article is similar to the appearance in our patients.)

Amorphous extracellular material consisting of fine filaments with a specific periodicity of 4 to $5 \mathrm{~nm}$ has been described as a pathological feature of XRS. ${ }^{6}$ These filaments are supposed to be the product of defective Müller cells.

It is conceivable that the fundus albipunctatuslike flecks in XRS also represent accumulations of this kind of amorphous material. However, there are two inconsistencies in this theory: (1) why is the fundus albipunctatus-like appearance so rarely noted while the deposition of abnormal amorphous material is a regular event in XRS?; (2) the localisation of the punctate white flecks that is, around the macular area, is not an area in which schisis usually develops.

The first inconsistency could be explained by the assumption that the albipunctatus-like flecks are a transient phenomenon (such as Boisdequin et al propose) occurring only in small children. Our two patients were very young, as were three patients of Boisdequin et al, including two brothers. One of Boisdequin et al's patients was about 40 years old, but this patient lacked a family history, the typical star-shaped maculopathy, and peripheral retinoschisis, while no electroretinography was performed.

For the second inconsistency we have no suitable explanation.

It is likely that the punctate lesions in XRS have often passed unnoticed. In the article by Greven $e t a l$ the picture of their patient 4 (21 months old) clearly shows the punctations, but they are not mentioned in the text. On the other hand, when the albipunctatus-like aspect in a particular male patient is noted, but not recognised as a possible manifestation of XRS, diagnostic confusion might ensue. Therefore we wish to report this symptom of XRS. To understand its nature and origin, future observations, both clinical and histopathological, will be important.

MJVS thanks Professor L N Went for his support and critical comments.

1 Deutman AF. Sex-linked juvenile retinoschisis. In: The hereditary dystrophies of the posterior pole of the eye. Assen: Van Gorcum \& Comp NV, 1971: 48-99.

2 Boisdequin D, Croughs P, Regnier P. Le Rétinoschisis. Bull Soc belge d'Ophtalmol 1981; 198: 51-89.

3 Marmor MF. Longterm follow-up of the physiologic abnormalities and fundus changes in fundus albipunctatus. malities and fundus changes
Ophthalmology 1990; 97: 380-4.

4 Miyake Y, Shiroyama N, Sugita S, Horiguchi M, Yagasaki K. Fundus albipunctatus associated with cone dystrophy. Brf Ophthalmol 1992; 76: 375-9.

5 Miyake Y, Miyake S, Yanagida K, Kanda T. X-chromosomal congenital retinoschisis. Its fundus polymorphism and visual function. Acta Soc Ophthalmol fpn 1981; 85: 97-112.

6 Condon GP, Brownstein S, Wang NS, Kearns AF, Ewing CC. Congenital hereditary (juvenile $\mathrm{X}$-linked) retinoschisis. Histopathologic and ultrastructural findings in three eyes. Arch Ophthalmol 1986; 104: 576-83.

7 Greven CM, Moreno RJ, Tasman W. Unusual manifestations of X-linked retinoschisis Trans Am Ophthalmol Soc 1990; 88: 